Nervenarzt 2021 · 92:975-976

https://doi.org/10.1007/s00115-021-01177-y

Angenommen: 27. Juli 2021

(c) Springer Medizin Verlag $\mathrm{GmbH}$, ein Teil von Springer Nature 2021

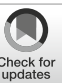

\section{Autor}
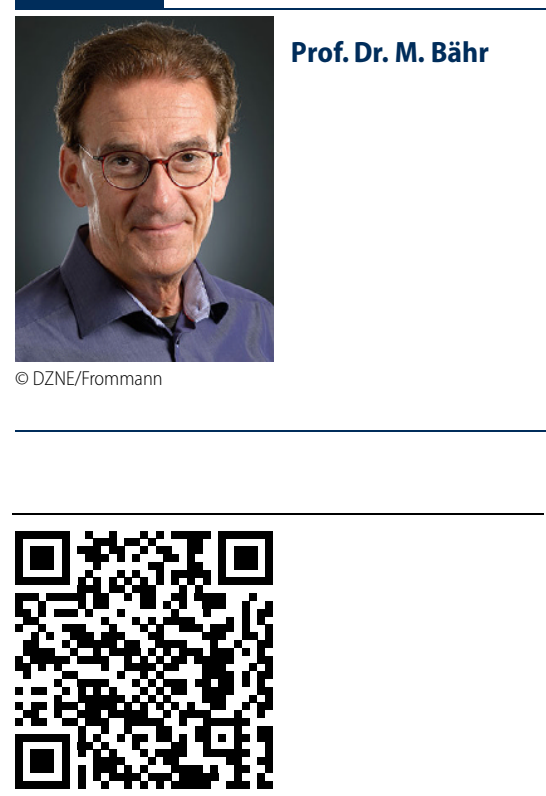

QR-Code scannen \&Beitrag online lesen

\title{
Über die systemmedizinische Analyse zur personalisierten Neurologie
}

\author{
M. Bähr \\ Klinik für Neurologie, Universitätsmedizin Göttingen, Göttingen, Deutschland
}

Traditionell folgen die klinischen Fachdisziplinen einer organbezogenen Betrachtungsweise. So beschäftigen sich die Kardiologen mit dem Herz, die Nephrologen mit der Niere und die Hepatologen mit der Leber. Wir als Neurologen mussten schon immer einen etwas umfassenderen Blick auf unsere Patienten werfen, da wir uns nicht nur mit Gehirn- und Rückenmarkserkrankungen beschäftigen, sondern u.a. auch mit neuromuskulären Krankheiten und Veränderungen des Immunsystems. Außerdem werden viele Erkrankungen des Nervensystems durch eine Pathologie anderer Organe ausgelöst, wie z.B. Schlaganfall, Hirnmetastasen oder paraneoplastische und autoimmune Syndrome. An den Schnittstellen zu anderen Organsystemen gab und gibt es wichtige Kooperationen. Ein stringent organübergreifender Denkansatz in Klinik und Forschung ist dennoch die Ausnahme (geblieben). Mit dem diesjährigen Kongressthema wollen wir einen Schwerpunkt auf die Systemmedizin legen und gleichzeitig deutlich machen, dass dadurch eine spezifischere und somit personalisierte diagnostische und therapeutische Vorgehensweise möglich wird: Wenn man Organe wie Herz und Gehirn nicht mehr getrennt betrachtet, sondern als eng vernetzte, miteinander kommunizierende Organsysteme, die einander beeinflussen, entsteht ein erweitertes und differenzierteres Bild wie S. Deking und J. Liman in ihrem Beitrag berichten. In der Konsequenz führt das zur Definition neuer Krankheitsentitäten bzw. es ergeben sich neue Hypothesen bezüglich deren Pathophysiologie. So ist die Takotsubo-Kardiomyopathie, auch „Syndrom des gebrochenen Herzens" genannt, wahrscheinlich keine primäre Herzerkrankung, sondern eine Dys- regulation des zentralen autonomen Netzwerks in Kombination mit einer Adrenalinhypersensitivität. Die systemmedizinische Betrachtungsweise ermöglicht uns somit eine neue Art von Präzisionsmedizin.

\section{》) Die Neurologie ist dazu prädestiniert, Fächergrenzen aufzulösen}

Wir glauben, dass die Neurologie als Fach prädestiniert ist, einen entsprechenden organübergreifenden Ansatz nicht nur zusammen mit Kardiologen zu verfolgen, sondern mit fast allen anderen großen Fächern. Wir Neurologen sind seit jeher darauf ausgerichtet, andere Fachdisziplinen konsiliarisch zu beraten und mit diesen zu interagieren. Daher existieren bereits viele Überschneidungsbereiche. Zum anderen haben wir sehr gute Möglichkeiten, die Fächergrenzen aufzulösen - sowohl in der Forschung als auch in der Versorgung: In vielen Stroke-Units etwa arbeiten bereits standardmäßig Kardiologen, die die kardiale Diagnostik direkt vor Ort ausführen können. Ähnliche Schnittstellen können wir uns in Zukunft auch zu anderen Fächern wie der Gastroenterologie, der Immunologie oder der Onkologie vorstellen.

In der Neuroimmunologie ist die präklinische und klinische Forschung ohne enge Kooperation und Interaktion mit Immunologen, Rheumatologen, Infektiologen, Toxikologen, Gastroenterologen und vielen anderen Fachdisziplinen kaum denkbar. Dies wird auch in dem umfassenden Übersichtsartikel von $R$. Linker und $R$. Gold deutlich: Zum einen konnten auf der Basis eines detaillierteren Verständnisses der komplexen Interaktionen verschiedener Immunzellen (T-Zellen, B-Zel- 
len, Plasmazellen, Mikroglia etc.) und deren Subtypen völlig neue orale, aber auch antikörperbasierte Therapiekonzepte entwickelt werden. Hier steht nun der Schritt zur individualisierten bzw. personalisierten Therapie an. Gleichzeitig wurde aber deutlich, dass Umweltfaktoren und systemisch wirksame Einflüsse wie Vitamin-DStatus, UV-Exposition, Nikotingenuss, hohe Salzzufuhr, übermäßiger Fettkonsum sowie Übergewicht den Krankheitsverlauf bei Multipler Sklerose stark beeinflussen. Diese Erkenntnisse eröffnen neue Wege für die personalisierte Präzisionsdiagnostik und individualisierte (Begleit-)Therapie, z. T. in Kooperation mit angrenzenden Fachdisziplinen wie der Gastroenterologie oder Nephrologie.

\section{I) Mit einer hochspezialisierten Diagnostik kann eine personalisierte Therapie umgesetzt werden}

In dem Beitrag von M. Platten, L. Bunse und W. Wick zur Neuroonkologie werden die Fortschritte in der Immunologie und die Verfügbarkeit neuer Therapeutika wie z. B. von Immun-Checkpoint-Inhibitoren dargestellt. Diese und andere neue Therapieansätze basieren auf der Entwicklung innovativer Vakzinen, der gezielten Modifikation des Hirntumormikromilieus sowie der Herstellung genetisch veränderter Lymphozyten zur adaptiven Zelltherapie. Es ist bereits absehbar, dass vor dem Hintergrund einer eingehenden hochspezialisierten Diagnostik u.a. mithilfe genetischer Subtypisierung von Tumorzellen und genauer Kenntnis des Host-Milieus eine personalisierte Therapie umgesetzt werden kann. Ohne die enge Verbindung zur onkologischen Grundlagenforschung und Translation in der Hämatoonkologie, Immunologie und Strahlentherapie wären auch hier die beschriebenen Innovationen kaum möglich gewesen.

Zusammenfassend betreffen Innovationen in der Neurologie zunehmend Schnittstellen zu anderen Fächern, die eine organübergreifende systemmedizinische Betrachtungsweise erfordern. Gleichzeitig eröffnen sich dadurch neue diagnostische und therapeutische Möglichkeiten, die in Zukunft eine personalisiertere und somit spezifischere Vorgehensweise ermöglichen könnten.

Prof. Dr. M. Bähr

\section{Korrespondenzadresse}

\section{Prof. Dr. M. Bähr}

Klinik für Neurologie, Universitätsmedizin

Göttingen

Robert-Koch-Str. 40, 37075 Göttingen,

Deutschland

mbaehr@gwdg.de

Interessenkonflikt. M. Bähr gibt an, dass kein Interessenkonflikt besteht.

\section{MEditorial}

\section{Hilfestellungen für den Editorial Manager}

Das Einreichungs- und Begutachtungssystem Ihrer Zeitschrift

Sowohl für die ganz alltäglichen Fragen in der Handhabung des Editorial Managers als auch für spezielle Problematiken finden Sie auf www.springermedizin.de/editorialmanager eine Vielzahl an Handreichungen, die Ihnen die Arbeit als Gutachter*in, Autor*in oder Herausgeber*in erleichtern.

Über Videos, einseitige Schritt-für-SchrittAnleitungen oder ein umfangreiches Manual werden Sie durch die einzelnen Punkte geführt, wie:

- Wie reiche ich ein Manuskript ein?

- Wie finde ich passende Gutachter*innen?

- Wie lade ich Gutachter*innen ein?

- Wie nehme ich ein Gutachten an bzw. lehne es ab?

- Wo erkenne ich, in welchem Status ein Mansukript ist?

- Wie ändere ich meine persönlichen Informationen?

- Wo kann ich meinen Urlaub eintragen?

Zugang auch über QR-Code:



